\title{
Electronic and Lattice Dynamical Properties of the Iron-Based Superconductors LiFeAs and NaFeAs
}

\author{
R. A. Jishi and H. M. Alyahyaei \\ Department of Physics, California State University, Los Angeles, CA 90032, USA \\ Correspondence should be addressed to R. A. Jishi, raljish@exchange.calstatela.edu \\ Received 3 March 2009; Accepted 17 June 2009 \\ Academic Editor: Igor Mazin
}

Copyright ( $) 2010$ R. A. Jishi and H. M. Alyahyaei. This is an open access article distributed under the Creative Commons Attribution License, which permits unrestricted use, distribution, and reproduction in any medium, provided the original work is properly cited.

\begin{abstract}
The electronic structure and lattice vibrational frequencies of the newly discovered superconductors, LiFeAs and NaFeAs, are calculated within density functional theory. We show that, in the vicinity of the Fermi energy, the density of states is dominated by contributions from Fe $3 \mathrm{~d}$ states. We also calculate the electron-phonon coupling strength and show that it is too weak to account for the observed values of the superconducting transition temperatures. This seems to indicate that the iron-based superconductors are not of the conventional type.
\end{abstract}

\section{Introduction}

A new class of layered, high- $T_{c}$ superconductors has been recently discovered. Kamihara et al. [1] reported a superconducting transition temperature $T_{c}=26 \mathrm{~K}$ in fluorinedoped LaFeAsO. Shortly afterwards, it was found that under pressure $T_{c}$ increased to $43 \mathrm{~K}$ [2]. Replacement of lanthanum with other rare earth metals gave a series of superconducting compounds $\mathrm{ReFeASO}_{1-\mathrm{x}} \mathrm{F}_{\mathrm{x}}$, where $\mathrm{Re}=\mathrm{Ce}, \mathrm{Pr}, \mathrm{Nd}, \mathrm{Sm}$, or $\mathrm{Gd}$, with transition temperatures close to or exceeding $50 \mathrm{~K}$ [3-8]. Oxygen deficient samples were also synthesized and found to superconduct at $55 \mathrm{~K}$ [9-11]. Hole doping, through the partial substitution of La with $\mathrm{Sr}$, or Gd with Th, was also found to yield superconducting compounds [12, 13]. Using-high pressure techniques, it was possible to increase the concentration of the F-dopant [14] and to synthesize superconducting compounds where $\mathrm{La}$ is replaced by the late rare earth elements $\mathrm{Tb}$ and $\mathrm{Dy}[15,16]$. The parent compound $\mathrm{ReFeAsO}$ is a layered compound consisting of a stack of alternating $\mathrm{ReO}$ and FeAs layers. Each ReO layer consists of an O-sheet surrounded by two Re sheets. Similarly, each FeAs layer consists of an Fe-sheet surrounded by two As sheets such that each Fe atom is tetrahedrally coordinated to four As atoms. Neutron diffraction measurements [1720] establish that the Fe magnetic moments adopt a collinear antiferromagnetic (c-AFM) order whereby ferromagnetic chains are coupled antiferromagnetically along the direction orthogonal to the chains.

Superconductivity was also discovered in a second class of compounds containing FeAs layers, namely, $\mathrm{AFe}_{2} \mathrm{As}_{2}$, where $\mathrm{A}$ is an alkaline earth metal. Hole doping, by partial replacement of $\mathrm{A}$ with alkali metals, results in superconducting compounds with $T_{c}$ reaching $38 \mathrm{~K}$ in $\mathrm{BaFe}_{2} \mathrm{As}_{2}$ and $\mathrm{SrFe}_{2} \mathrm{As}_{2}$ [21-25]. Partial substitution of Fe with Co was also shown to give a superconducting compound with $T_{c}=22 \mathrm{~K}$ [26]. Similarly to the first class, in the parent compounds the Fe magnetic moments in this second class have a collinear AFM order with a spin-stripes pattern [27-29]. In both classes, the Fe magnetic moments in the parent compounds exhibit magnetic order, at low temperature, which disappears upon doping, making way for the emergence of superconductivity. This leads to the reasonable belief that strong electronic correlations are important in these systems, and that superconductivity in these compounds is somehow connected to magnetic fluctuations [30-42]. Indeed, the electron-phonon coupling in LaOFeAs was estimated to be too small [43] to give rise to superconductivity within the conventional BCS formulation. [44]

Recently, a third class of iron-based superconductors was discovered. LiFeAs and NaFeAs were found to superconduct below $18 \mathrm{~K}$ and $9 \mathrm{~K}$, respectively, [45-48]. It turns out that in these two compounds no magnetic order is detected at 
all temperatures. In some sense, these two compounds are important with regards to understanding the mechanism of superconductivity in iron-based superconductors. The absence of spin density wave (SDW) transition, on the one hand, and the relatively low $T_{c}$ in comparison with the first two classes of iron-based superconductors, on the other hand, make these two compounds possible candidates for being conventional BCS superconductors.

Band structure calculations, using local density approximation (LDA) within density functional theory (DFT), were recently reported for $\mathrm{LiFeAs}[49,50]$. It was found that LiFeAs is semimetallic, and that the density of states (DOSs) near the Fermi level is dominated by the Fe $3 \mathrm{~d}$ states. Thus, the electronic structure of stoichiometric LiFeAs is similar to that of the parent compounds of the first class, with a hole cylinder at the Brillouin zone (BZ) center, electron cylinders at the BZ corners, and an electronic DOS that decreases strongly with increasing energy in the vicinity of the Fermi energy.

In this work we report DFT calculations of the electronic and lattice properties of LiFeAs and NaFeAs. In particular, we calculate the electron-phonon coupling strength and show that it is too weak to account for the superconducting transition temperatures observed in these compounds. Our calculations, together with previous calculations [44] of the electron-phonon coupling strength in LaOFeAs, seem to indicate that iron-based superconductors are not of the conventional type.

\section{Method}

The electronic structure calculations are carried out using the all-electron full-potential linear augmented plane wave (FP-LAPW) method as implemented in WIEN2K code [51]. The exchange-correlation potential was calculated using the generalized gradient approximation (GGA) as proposed by Pedrew, Burke, and Ernzerhof (PBE) [52]. The radii of the muffin-tin spheres for the various atoms were chosen so that the neighboring spheres almost touch each other. We set the parameter $R_{\mathrm{MT}} K_{\max }=7$, where $R_{\mathrm{MT}}$ is the smallest muffintin radius, and $K_{\max }$ is a cutoff wave vector. The valence wave functions inside the muffin-tin spheres are expanded in terms of spherical harmonics up to $I_{\max }=10$, and in terms of plane waves with a wave vector cutoff $K_{\max }$ in the interstitial region. The charge density is Fourier expanded up to $G_{\max }=$ $13 a_{0}^{-1}$, where $a_{0}$ is the Bohr radius. Convergence of the selfconsistent field calculations is attained with a total energy convergence tolerance of $0.01 \mathrm{mRy}$.

The calculation of the frequencies of the vibrational modes and the electron-phonon coupling parameter was performed using ultrasoft pseudopotentials and an expansion of the wave function of the valence electrons in terms of plane waves, with an energy cutoff of 30 Rydbergs [53]. The charge density is Fourier expanded with an energy cutoff of 400 Rydbergs. The dynamical matrices and the electronphonon coupling parameter are calculated using DFT in the linear response approximation. In calculating the phonon frequencies, the electronic integration is carried out using a uniform mesh of $N_{k}=4 \times 4 \times 4 k$-points in the Brillouin

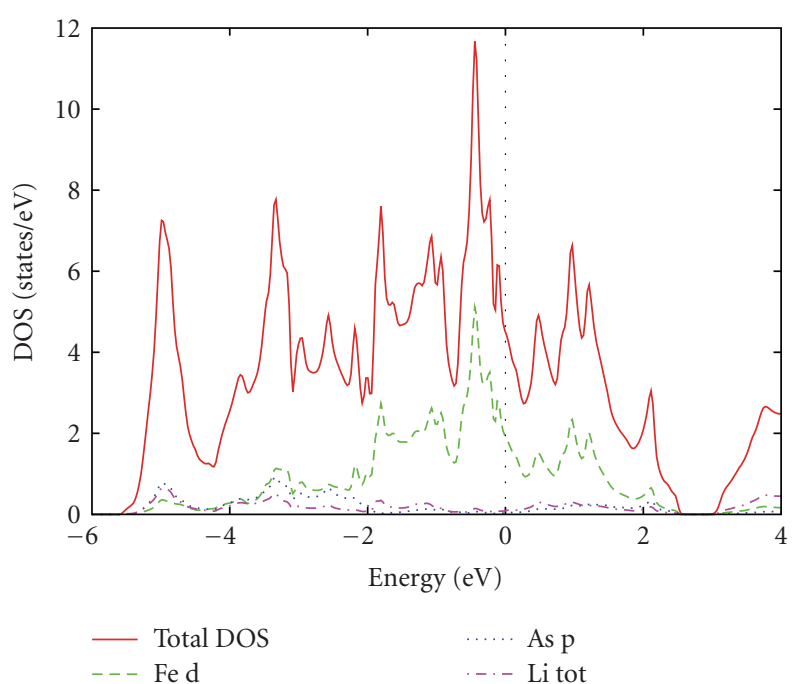

FIgURE 1: Density of states (DOSs) in LiFeAs. Both the total and atomic DOSs are shown. The Fermi energy is the zero energy. Near the Fermi energy, the DOS is dominated by the Fe $3 \mathrm{~d}$ states.

zone. On the other hand, the electronic density of states, used in computing the electron-phonon coupling parameter $\lambda$, is calculated using a finer mesh of $16 \times 16 \times 16 k$-points. The value of $\lambda$ is obtained by averaging over a uniform mesh of $N_{q}=4 \times 4 \times 4$ phonon momentum $q$-points. The phonon dispersion curves are obtained by Fourier interpolation of the dynamical matrices computed on the $\mathrm{N}_{\mathrm{q}}$ points mesh.

In the electronic and lattice calculations, the experimental values of the low-temperature lattice constants and atomic positions [45-48] are used. For both compounds, the crystal is tetragonal with space group P4/nmm. In LiFeAs, the lattice constants are $a=3.76982 \AA, c=6.30693 \AA$, whereas in $\mathrm{NaFeAs}, a=3.94729 \AA$, and $c=6.99112 \AA$.

\section{Results and Discussion}

Our results for the electronic structure calculations for LiFeAs and NaFeAs are summarized in Figures 1 and 2, respectively, where the electronic density of states (DOSs) is displayed. For LiFeAs, our calculated DOS is similar to that reported earlier $[49,50]$. In both LiFeAs and NaFeAs, the DOS plots show some generic features that are common to the parent compounds of the iron-based superconductors: a DOS that is dominated by the Fe $3 d$ states in the vicinity of the Fermi energy, with only a small contribution from the As and alkali metal states and that is strongly decreasing with energy near the Fermi energy.

It should be noted that in a unit cell of LiFeAs, for example, there are two Li, two Fe, and two As atoms. Thus to get the total atomic DOS, the values of the atomic DOS shown in 1 should be multiplied by 2 . The total DOS is the sum of the total atomic DOS and the DOS in the interstitial region.

Since the DOS at the Fermi energy, $N\left(\mathrm{E}_{\mathrm{F}}\right)$, is $\sim 4$ states/eV in both LiFeAs and NaFeAs, which is not very small, and because of the relatively lower $T_{c}$ compared with the 


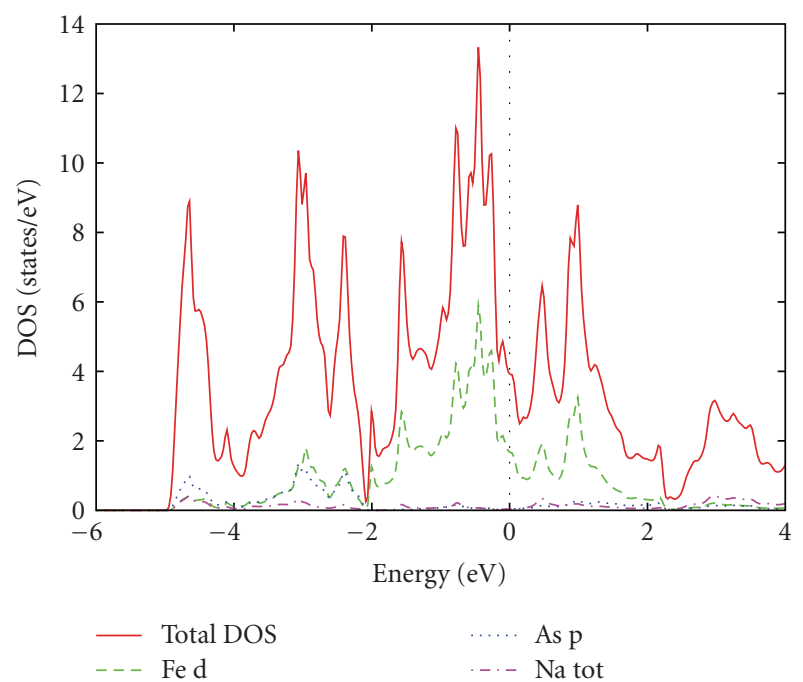

Figure 2: Density of states (DOSs) in NaFeAs. Both the total and atomic DOSs are shown. The Fermi energy is the zero energy. Near the Fermi energy, the DOS is dominated by the Fe $3 \mathrm{~d}$ states.

other iron-based superconductors, one may wonder whether electron-phonon coupling may lie behind the mechanism for superconductivity in LiFeAs and NaFeAs. This notion may be given more credence by the observation that a sodium atom is about three times more massive than a lithium atom, so that if the attractive electron-electron interaction is mediated by the alkali-metal atomic vibrations, then this difference in the mass could explain the difference in the values of $T_{c}$ between the two compounds via the well-known isotope effect.

To test this idea, we carried out a calculation of the phonon dispersion curves and the electron-phonon coupling strength in these compounds. Since the crystallographic point group in LiFeAs and NaFeAs is $D_{4 \mathrm{~h}}$, the vibrational modes at $\Gamma$, the $\mathrm{BZ}$ center, are decomposed according to the following irreducible representations:

$$
\Gamma_{\text {phonon }}=2 A_{1 g}+B_{1 g}+3 E_{g}+3 A_{2 u}+3 E_{u} .
$$

The acoustic modes, with vanishing frequency at $\Gamma$, the $\mathrm{BZ}$ center, transform according to the $A_{2 \mathrm{u}}$ and $E_{\mathrm{u}}$ irreducible representations. Excluding the acoustic modes, we are left with 15 modes with nonzero frequencies; among these, the symmetric ones are Raman-active, while the antisymmetric modes are infrared-active. The calculated frequencies of the Raman- and infrared-active modes at the $\Gamma$ point of the BZ are given in Table 1. The phonon dispersion curves in LiFeAs, plotted along high symmetry directions in the BZ, are shown in Figure 3, and the corresponding curves in NaFeAs are given in Figure 4. Our results for the phonon frequencies at the BZ center may be checked by Raman scattering and infrared absorption experiments, while the phonon dispersion curves may be checked by neutron scattering measurements.

We calculated the electron-phonon coupling parameter $\lambda$ and found it to be 0.29 and 0.27 for LiFeAs and $\mathrm{NaFeAs}$, respectively. For conventional superconductors,

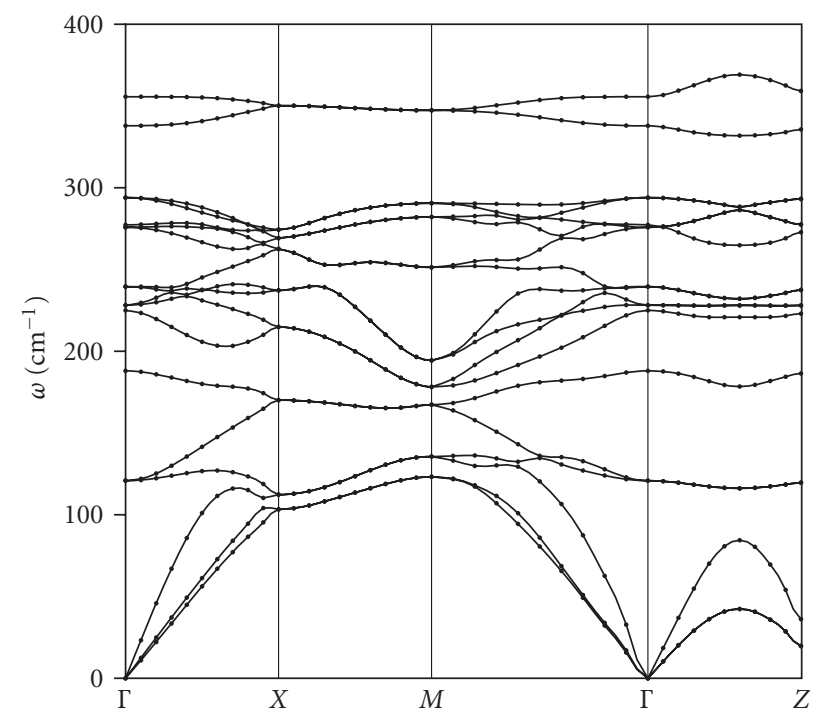

Figure 3: Phonon dispersion curves in LiFeAs, plotted along high symmetry directions of the Brillouin zone.

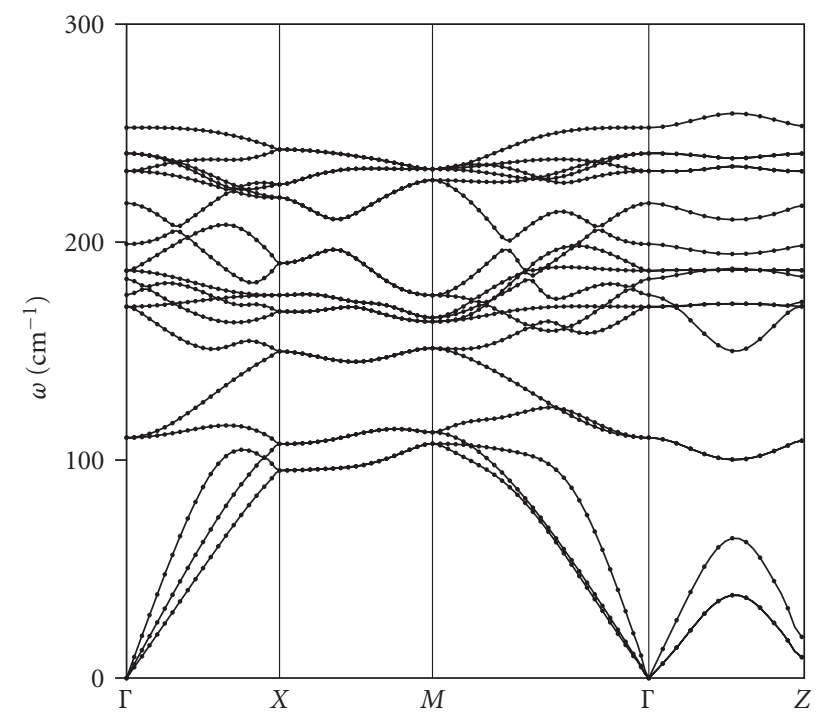

Figure 4: Phonon dispersion curves in NaFeAs, plotted along high symmetry directions of the Brillouin zone.

where the attractive electron-electron interaction is mediated by phonons, the transition temperature is given by the Allen and Dynes [54] modified McMillan's formula [55]

$$
T_{c}=\frac{\omega_{\log }}{1.2} \exp \left[\frac{-1.04(1+\lambda)}{\lambda-\mu^{*}(1+0.62 \lambda)}\right]
$$

where $\omega_{\log }$ is the logarithmic average phonon frequency, expressed in degrees Kelvin, and $\mu^{*}$ is the Coulomb pseudopotential parameter, usually taken to be $\sim 0.13$. Since $\omega_{\log }$ in LiFeAs and NaFeAs is of the order of $100 \mathrm{~K}$, the resulting value of $T_{c}$ is much less than $1 \mathrm{~K}$. We conclude that the electron-phonon coupling is too weak to account for superconductivity in this class of iron-based superconductors. 
TABLE 1: The calculated frequencies, in $\mathrm{cm}^{-1}$, of the Raman- and infrared-(IR-) active modes in LiFeAs and NaFeAs. The modes are classified by the irreducible representations (irreps) according to which they transform.

\begin{tabular}{|c|c|c|c|c|c|c|c|}
\hline & & & & $/ \mathrm{cm}^{-1}$ & & & \\
\hline \multirow{2}{*}{ LiFeAs } & Ramman & $121\left(E_{g}\right)$ & $188\left(A_{1 g}\right)$ & $225\left(B_{1 g}\right)$ & $240\left(E_{g}\right)$ & $294\left(E_{g}\right)$ & $356\left(A_{1 g}\right)$ \\
\hline & IR & $228\left(E_{u}\right)$ & $276\left(E_{u}\right)$ & $277\left(A_{2 u}\right)$ & $338\left(A_{2 u}\right)$ & & \\
\hline \multirow{2}{*}{ NaFeAs } & Ramman & $110\left(E_{g}\right)$ & $176\left(A_{1 g}\right)$ & $187\left(E_{g}\right)$ & $199\left(A_{1 g}\right)$ & $218\left(B_{1 g}\right)$ & $241\left(E_{g}\right)$ \\
\hline & IR & $170\left(E_{u}\right)$ & $183\left(A_{u}\right)$ & $233\left(E_{u}\right)$ & $253\left(A_{2 u}\right)$ & & \\
\hline
\end{tabular}

There are some puzzling questions that beset this third class of iron-based superconductors. In the parent compounds of the first two classes of iron-based superconductors, magnetic order is established at low temperatures, where the Fe magnetic moments adopt a collinear antiferromagnetic (c-AFM) order with a stripe-like pattern; this is unequivocally confirmed by both neutron diffraction measurements [17-20] and DFT calculations [31-39]. In the first class, it is only upon electron doping through the replacement of a small percentage of oxygen atoms with fluorine atoms, or the removal of a small percentage of oxygen atoms, that the magnetic order is suppressed and superconductivity emerges. In the second class of iron-based superconductors, magnetic order is suppressed by hole doping through the replacement of some alkaline earth atoms with alkali atoms. To better understand the situation in the third class of iron-based superconductors, we carried out spin-polarized DFT calculations on stoichiometric LiFeAs and $\mathrm{NaFeAs}$, in addition to the calculations reported above for the nonmagnetic phases of these compounds. We considered, within GGA, various spin arrangements on the Fe sites. Similarly to the case of the first two classes, we find that the c-AFM phase, with a spin-stripes pattern, is indeed the lowest energy phase. Within GGA, the energy of the c-AFM phase in LiFeAs is lower than the AFM phase by $0.081 \mathrm{eV}$ per $\mathrm{Fe}$ atom $(\mathrm{eV} / \mathrm{Fe})$, lower than the ferromagnetic (FM) phase by $0.085 \mathrm{eV} / \mathrm{Fe}$, and lower than the nonmagnetic phase by $0.123 \mathrm{eV} / \mathrm{Fe}$. For NaFeAs, the energy of the c-AFM phase is lower than the AFM phase by $0.048 \mathrm{eV} / \mathrm{Fe}$, lower than the FM phase by $0.205 \mathrm{eV} / \mathrm{Fe}$, and lower than the nonmagnetic phase by $0.182 \mathrm{eV} / \mathrm{Fe}$. The calculated magnetic moment on an $\mathrm{Fe}$ site is $2.18 \mu_{\mathrm{B}}$ in the case of NaFeAs, and $1.76 \mu_{\mathrm{B}}$ in the case of LiFeAs, where $\mu_{\mathrm{B}}$ is the Bohr magneton. Thus, according to our DFT calculations, stoichiometric LiFeAs and NaFeAs should be similar to the parent compounds of the first two classes, and they should not superconduct; instead, at low temperature, the stoichiometric compounds should display magnetic order. Deviations from stoichiometry, on the other hand, may suppress the magnetic order, making way for superconductivity, just like doping does in the first two classes of iron-based compounds. In the case of LiFeAs, it is indeed the case that the synthesized superconducting compounds were not stoichiometric, the chemical formula being $\mathrm{Li}_{1-x} \mathrm{FeAs}$ [47]. The situation is less clear in the case of NaFeAs [48], but we believe, on the basis of our spinpolarized calculations and the absence of any detectable magnetic order at low temperatures, that the synthesized $\mathrm{NaFeAs}$ samples may also be nonstoichiometric.
We should note, however, that our results on the magnetic structure of the third class of compounds are only tentative. One problem is that the calculated magnetic moment per $\mathrm{Fe}$ site is too large in comparison with the experimental value in $\mathrm{LaFeAsO}$, where the Fe moments also adopt c-AFM order, albeit with a magnetic moment of only $0.36 \mu_{\mathrm{B}}$ [17]. It has been shown by various groups [5658] that even though the Fe moments adopt the stripeAFM order in the undoped LaFeAsO compound, yet the stabilization energy and the value of the $\mathrm{Fe}$ magnetic moment are sensitive to the type of exchange-correlation functional employed in the calculation, and to the position of the As atom. At this point, all we can say is that DFT calculations within GGA using PBE exchange correlation potential suggest that stoichiometric LiFeAs and NaFeAs compounds will display magnetic order at low temperatures.

\section{Conclusions}

In conclusion, we have presented the results of electronic structure calculations on LiFeAs and NaFeAs, members of a new class of superconducting compounds. In similarity to other iron-based superconductors, the density of states in the vicinity of the Fermi energy is found to be dominated by contributions from the $\mathrm{Fe} 3 \mathrm{~d}$ states. We have also calculated the Raman and infrared phonon frequencies at the Brillouin zone center, as well the phonon dispersion curves along high symmetry directions in the Brillouin zone. We have evaluated the electron-phonon coupling parameter in LiFeAs and NaFeAs and found its value to be too small to account for the observed superconducting transition temperatures in these compounds. Our results, taken together with previous estimates of the electron-phonon coupling strength in LaFeAsO, seem to suggest clearly that iron-based superconductors are not of the conventional type, where the attractive electron-electron interaction is mediated by phonons.

\section{References}

[1] Y. Kamihara, T. Watanabe, M. Hirano, and H. Hosono, "Ironbased layered superconductor $\mathrm{La}\left[\mathrm{O}_{1-x} \mathrm{~F}_{x}\right] \mathrm{FeAs}(x=0.05$ 0.12) with Tc $=26 \mathrm{~K}$," Journal of the American Chemical Society, vol. 130, no. 11, pp. 3296-3297, 2008.

[2] H. Takahashi, K. Igawa, K. Arii, Y. Kamihara, M. Hirano, and $\mathrm{H}$. Hosono, "Superconductivity at $43 \mathrm{~K}$ in an iron-based layered compound $\mathrm{LaO}_{1-x} \mathrm{~F}_{x} \mathrm{FeAs}$," Nature, vol. 453, no. 7193, pp. 376-378, 2008. 
[3] G. F. Chen, Z. Li, D. Wu, et al., "Superconductivity at 41 $\mathrm{K}$ and its competition with spin-density-wave instability in layered $\mathrm{CeO}_{1-x} \mathrm{~F}_{x}$ FeAs," Physical Review Letters, vol. 100, no. 24, Article ID 247002, 2008.

[4] Z.-A. Ren, J. Yang, W. Lu, et al., "Superconductivity at $52 \mathrm{~K}$ in iron based $\mathrm{F}$ doped layered quaternary compound $\operatorname{Pr}\left[\mathrm{O}_{1-x} \mathrm{~F}_{x}\right]$ FeAs," Materials Research Innovations, vol. 12, no. 3, pp. 105106, 2008.

[5] Z.-A. Ren, J. Yang, W. Lu, et al., "Superconductivity in the iron-based F-doped layered quaternary compound $\mathrm{Nd}\left[\mathrm{O}_{1-x} \mathrm{~F}_{x}\right]$ FeAs," Europhysics Letters, vol. 82, no. 5, Article ID 57002, 2008.

[6] X. H. Chen, T. Wu, G. Wu, R. H. Liu, H. Chen, and D. F. Fang, "Superconductivity at $43 \mathrm{~K}$ in $\mathrm{SmFeAsO}_{1-x} \mathrm{~F}_{x}$," Nature, vol. 453, no. 7196, pp. 761-762, 2008.

[7] Z.-A. Ren, W. Lu, J. Yang, et al., "Superconductivity at $55 \mathrm{~K}$ in iron-based F-doped layered quaternary compound $\mathrm{Sm}\left[\mathrm{O}_{1-x} \mathrm{~F}_{x}\right]$ FeAs," Chinese Physics Letters, vol. 25, no. 6, pp. 2215-2216, 2008.

[8] P. Cheng, L. Fang, H. Yang, et al., "Superconductivity at $36 \mathrm{~K}$ in gadolinium-arsenide oxides $\mathrm{GdO}_{1-x} \mathrm{~F}_{x} \mathrm{FeAs}$," Science in China, Series G, vol. 51, no. 6, pp. 719-722, 2008.

[9] J. Yang, Z.-C. Li, W. Lu, et al., "Superconductivity at $53.5 \mathrm{~K}$ in GdFeAsO1- $\delta$," Superconductor Science and Technology, vol. 21, no. 8, Article ID 082001, 2008.

[10] G. Wu, Y. J. Yan, T. Wu, et al., "Superconductivity induced by oxygen deficiency in La0.85 Sr0.15 FeAsO1- $\delta$," Physical Review B, vol. 78, no. 9, Article ID 092503, 2008.

[11] Z.-A. Ren, G.-C. Che, X.-L. Dong, et al., "Superconductivity and phase diagram in iron-based arsenic-oxides $\mathrm{ReFeAsO} 1-\delta$ ( $\mathrm{Re}=$ rare-earth metal) without fluorine doping," Europhysics Letters, vol. 83, no. 1, Article ID 17002, 2008.

[12] H.-H. Wen, G. Mu, L. Fang, H. Yang, and X. Zhu, "Superconductivity at $25 \mathrm{~K}$ in hole-doped $\left(\mathrm{La}_{1-x} \mathrm{Sr}_{x}\right) \mathrm{OFeAs}$," Europhysics Letters, vol. 82, no. 1, Article ID 17009, 2008.

[13] C. Wang, L. Li, S. Chi, et al., "Thorium-doping-induced superconductivity up to $56 \mathrm{~K}$ in $\mathrm{Gd}_{1-x} \mathrm{Th}_{x} \mathrm{FeAsO}$," Europhysics Letters, vol. 83, no. 6, Article ID 67006, 2008.

[14] W. Lu, X.-L. Shen, J. Yang, et al., "Superconductivity at 41.0 $\mathrm{K}$ in the F-doped $\mathrm{LaFeAsO}_{1-x} \mathrm{~F}_{x}$," Solid State Communications, vol. 148, no. 3-4, pp. 168-170, 2008.

[15] J.-W. G. Bos, G. B. S. Penny, J. A. Rodgers, D. A. Sokolov, A. D. Huxley, and J. P. Attfield, "High pressure synthesis of late rare earth RFeAs $(\mathrm{O}, \mathrm{F})$ superconductors; $\mathrm{R}=\mathrm{Tb}$ and Dy," Chemical Communications, no. 31, pp. 3634-3635, 2008.

[16] L.-J. Li, Y.-K. Li, Z.-A. Ren, et al., "Superconductivity above $50 \mathrm{~K}$ in $\mathrm{Tb}_{1-x} \mathrm{Th}_{x} \mathrm{FeAsO}$," Physical Review B, vol. 78, no. 13, Article ID 132506, 2008.

[17] C. de la Cruz, Q. Huang, J. W. Lynn, et al., "Magnetic order close to superconductivity in the iron-based layered $\mathrm{LaO}_{1-x} \mathrm{~F}_{x} \mathrm{Fe}$ As systems," Nature, vol. 453, no. 7197, pp. 899$902,2008$.

[18] J. Zhao, Q. Huang, C. de la Cruz, et al., "Structural and magnetic phase diagram of $\mathrm{CeFeAsO}_{1-x} \mathrm{~F}_{x}$ and its relation to high-temperature superconductivity," Nature Materials, vol. 7, no. 12, pp. 953-959, 2008.

[19] J. Zhao, Q. Huang, C. de la Cruz, et al., "Lattice and magnetic structures of PrFeAsO, PrFeAsO0.85F 0.15, and PrFeAsO0.85," Physical Review B, vol. 78, no. 13, Article ID 132504, 2008.

[20] Y. Qiu, W. Bao, Q. Huang, et al., "Crystal structure and antiferromagnetic order in $\mathrm{NdFeAsO}_{1-x} \mathrm{~F}_{x}(x=0.0$ and 0.2 ) superconducting compounds from neutron diffraction measurements," Physical Review Letters, vol. 101, no. 25, Article ID 257002, 2008.
[21] M. Rotter, M. Tegel, and D. Johrendt, "Superconductivity at $38 \mathrm{~K}$ in the iron arsenide $\left(\mathrm{Ba}_{1-x} \mathrm{~K}_{x}\right) \mathrm{Fe}_{2} \mathrm{As}_{2}$," Physical Review Letters, vol. 101, no. 10, Article ID 107006, 2008.

[22] G.-F. Chen, Z. Li, G. Li, et al., "Superconductivity in holedoped $\left(\mathrm{Sr}_{1-x} \mathrm{~K}_{x}\right) \mathrm{Fe}_{2} \mathrm{As}_{2}$," Chinese Physics Letters, vol. 25, no. 9 , pp. 3403-3405, 2008.

[23] K. Sasmal, B. Lv, B. Lorenz, et al., "Superconducting febased compounds $\left(\mathrm{A}_{1-x} \mathrm{Sr}_{x}\right) \mathrm{Fe}_{2} \mathrm{As}_{2}$ with $\mathrm{A}=\mathrm{K}$ and $\mathrm{Cs}$ with transition temperatures up to $37 \mathrm{~K}$," Physical Review Letters, vol. 101, no. 10, Article ID 107007, 2008.

[24] G. Wu, R. H. Liu, Y. J. Yan, et al., "Transport properties and superconductivity in $\mathrm{Ba1}-\mathrm{xM} \mathrm{xFe}_{2} \mathrm{As}_{2}(\mathrm{M}=\mathrm{La}$ and $\mathrm{K})$ with double FeAs layers," Europhysics Letters, vol. 84, no. 2, Article ID 27010, 2008.

[25] N. Ni, S. L. Bud'ko, A. Kreyssig, et al., "Anisotropic thermodynamic and transport properties of single-crystalline $\mathrm{Ba}_{1-x} \mathrm{~K}_{x} \mathrm{Fe}_{2} \mathrm{As}_{2}$ ( $x=0$ and 0.45)," Physical Review B, vol. 78, no. 1, Article ID 014507, 2008.

[26] A. S. Sefat, R. Jin, M. A. McGuire, B. C. Sales, D. J. Singh, and D. Mandrus, "Superconductivity at $22 \mathrm{~K}$ in co-doped $\mathrm{BaFe}_{2} \mathrm{As}_{2}$ crystals," Physical Review Letters, vol. 101, no. 11, Article ID 117004, 2008.

[27] Q. Huang, Y. Qiu, W. Bao, et al., "Neutron-diffraction measurements of magnetic order and a structural transition in the parent $\mathrm{BaFe}_{2} \mathrm{As}_{2}$ compound of FeAs-based high-temperature superconductors," Physical Review Letters, vol. 101, no. 25, Article ID 257003, 2008.

[28] K. Kitagawa, N. Katayama, K. Ohgushi, M. Yoshida, and M. Takigawa, "Commensurate itinerant antiferromagnetism in $\mathrm{BaFe}_{2} \mathrm{As}_{2}$ : 75As-NMR studies on a self-flux grown single crystal," Journal of the Physical Society of Japan, vol. 77, no. 11, Article ID 114709, 2008.

[29] Y. Su, P. Link, A. Schneidewind, et al., "Antiferromagnetic ordering and structural phase transition in $\mathrm{Ba}_{2} \mathrm{Fe}_{2} \mathrm{As}_{2}$ with $\mathrm{Sn}$ incorporated from the growth flux," Physical Review B, vol. 79, no. 6, Article ID 064504, 2009.

[30] D. J. Singh and M.-H. Du, "Density functional study of $\mathrm{LaFeAsO}_{1-x} \mathrm{~F}_{x}$ : a low carrier density superconductor near itinerant magnetism," Physical Review Letters, vol. 100, no. 23, Article ID 237003, 2008.

[31] K. Haule, J. H. Shim, and G. Kotliar, "Correlated electronic structure of $\mathrm{LaO}_{1-x} \mathrm{~F}_{x} \mathrm{FeAs}$," Physical Review Letters, vol. 100, no. 22, Article ID 226402, 2008.

[32] J. Dong, H. J. Zhang, G. Xu, et al., "Competing orders and spin-density-wave instability in $\mathrm{La}\left(\mathrm{O}_{1-x} \mathrm{~F}_{x}\right) \mathrm{FeAs}$," Europhysics Letters, vol. 83, no. 2, Article ID 27006, 2008.

[33] F. Ma and Z.-Y. Lu, "Iron-based layered compound LaFeAsO is an antiferromagnetic semimetal," Physical Review B, vol. 78, no. 3, Article ID 033111, 2008.

[34] C. Cao, P. J. Hirschfeld, and H.-P. Cheng, "Proximity of antiferromagnetism and superconductivity in $\mathrm{LaFeAsO}_{1-x} \mathrm{~F}_{x}$ : effective Hamiltonian from ab initio studies," Physical Review $B$, vol. 77, no. 22, Article ID 220506, 2008.

[35] T. Yildirim, "Origin of the $150-\mathrm{K}$ anomaly in LaFeAsO: competing antiferromagnetic interactions, frustration, and a structural phase transition," Physical Review Letters, vol. 101, no. 5, Article ID 057010, 2008.

[36] Z. -Y. Weng, "Hidden SDW order and effective low-energy theory for FeAs superconductors," Physica E: Low-dimensional Systems and Nanostructures, vol. 41, no. 7, pp. 1281-1284, 2009.

[37] F. Ma, Z.-Y. Lu, and T. Xiang, "Arsenic-bridged antiferromagnetic superexchange interactions in LaFeAsO," Physical Review $B$, vol. 78, no. 22, Article ID 224517, 2008. 
[38] Z. P. Yin, S. Lebègue, M. J. Han, B. P. Neal, S. Y. Savrasov, and W. E. Pickett, "Electron-hole symmetry and magnetic coupling in antiferromagnetic LaFeAsO," Physical Review Letters, vol. 101, no. 4, Article ID 047001, 2008.

[39] S. Ishibashi, K. Terakura, and H. Hosono, "A possible ground state and its electronic structure of a mother material (LaOFeAs) of new superconductors," Journal of the Physical Society of Japan, vol. 77, no. 5, Article ID 053709, 2008.

[40] Q. Si and E. Abrahams, "Strong correlations and magnetic frustration in the high Tc iron pnictides," Physical Review Letters, vol. 101, no. 7, Article ID 076401, 2008.

[41] C. Fang, H. Yao, W.-F. Tsai, J. Hu, and S. A. Kivelson, "Theory of electron nematic order in LaFeAsO," Physical Review B, vol. 77, no. 22, Article ID 224509, 2008.

[42] C. Xu, M. Müller, and S. Sachdev, "Ising and spin orders in the iron-based superconductors," Physical Review B, vol. 78, no. 2, Article ID 020501, 2008.

[43] L. Boeri, O. V. Dolgov, and A. A. Golubov, "Is $\mathrm{LaFeAsO}_{1-x} \mathrm{~F}_{x}$ an electron-phonon superconductor?" Physical Review Letters, vol. 101, no. 2, Article ID 026403, 2008.

[44] J. Bardeen, L. N. Cooper, and J. R. Schrieffer, "Theory of superconductivity," Physical Review, vol. 108, no. 5, pp. 11751204, 1957.

[45] M. J. Pitcher, D. R. Parker, P. Adamson, et al., "Structure and superconductivity of LiFeAs," Chemical Communications, no. 45, pp. 5918-5920, 2008.

[46] J. H. Tapp, Z. Tang, B. Lv, et al., "LiFeAs: an intrinsic FeAsbased superconductor with Tc $=18 \mathrm{~K}$," Physical Review B, vol. 78, no. 6, Article ID 060505, 2008.

[47] X. C. Wang, Q. Q. Liu, Y. X. Lv, et al., "The superconductivity at $18 \mathrm{~K}$ in LiFeAs system," Solid State Communications, vol. 148, no. 11-12, pp. 538-540, 2008.

[48] D. R. Parker, M. J. Pitcher, P. J Baker, et al., "Structure, antiferromagnetism and superconductivity of the layered iron arsenide NaFeAs," Chemical Communications Articles, pp. 2189-2191, 2009.

[49] I. A. Nekrasov, Z. V. Pchelkina, and M. V. Sadovskii, "Electronic structure of new LiFeAs high-Tc superconductor," JETP Letters, vol. 88, no. 8, pp. 543-545, 2008.

[50] D. J. Singh, "Electronic structure and doping in $\mathrm{BaFe}_{2} \mathrm{As}_{2}$ and LiFeAs: density functional calculations," Physical Review B, vol. 78, no. 9, Article ID 094511, 2008.

[51] P. Blaha, K. Schwarz, G. K. H. Madsen, D. Kavasnicka, and J. Luitz, WIEN2K, An Augmented Plane Wave + Local Orbitals Program for Calculating Crystal Properties, Technische Universität, Wien, Austria, 2001.

[52] J. P. Perdew, K. Burke, and M. Ernzerhof, "Generalized gradient approximation made simple," Physical Review Letters, vol. 77, no. 18, pp. 3865-3868, 1996.

[53] P. Giannozzi, et al., http://www.quantum-espresso.org.

[54] P. B. Allen and R. C. Dynes, "Transition temperature of strongcoupled superconductors reanalyzed," Physical Review B, vol. 12, no. 3, pp. 905-922, 1975.

[55] W. L. McMillan, "Transition temperature of strong-coupled superconductors," Physical Review, vol. 167, no. 2, pp. 331$344,1968$.

[56] I. I. Mazin, M. D. Johannes, L. Boeri, K. Koepernik, and D. J. Singh, "Problems with reconciling density functional theory calculations with experiment in ferropnictides," Physical Review B, vol. 78, no. 8, Article ID 085104, 2008.

[57] A. N. Yaresko, G.-Q. Liu, V. N. Antonov, and O. K. Andersen, "Interplay between magnetic properties and Fermi surface nesting in iron pnictides," Physical Review B, vol. 79, no. 14, Article ID 144421, 2009.
[58] S. Sharma, J. K. Dewhurst, S. Shallcross, et al., "Magneticproperties of $\mathrm{LaO}_{1-x} \mathrm{~F}_{x} \mathrm{FeAs}$," http://arxiv.org/abs/0810.4278. 

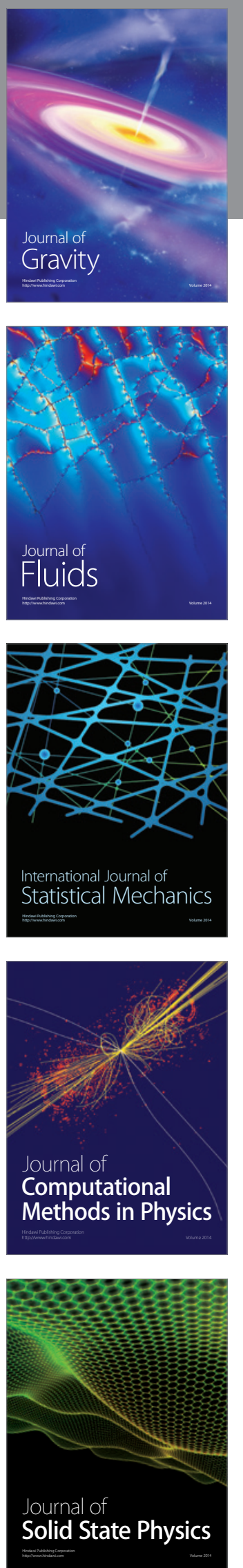

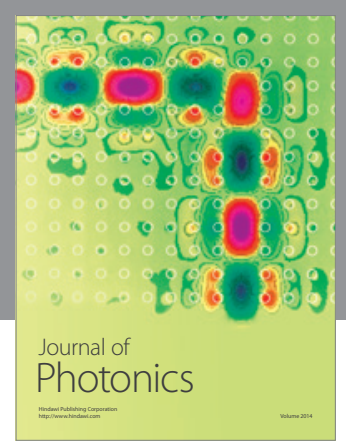

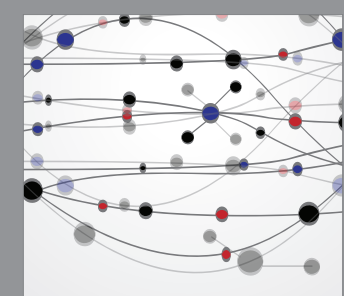

The Scientific World Journal
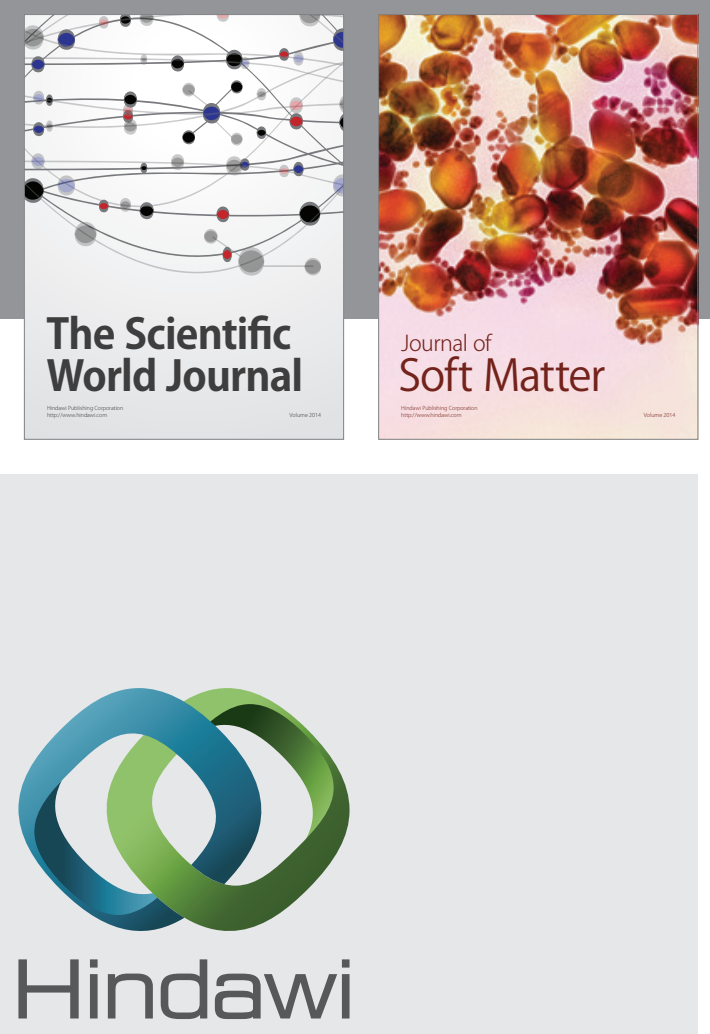

Submit your manuscripts at

http://www.hindawi.com
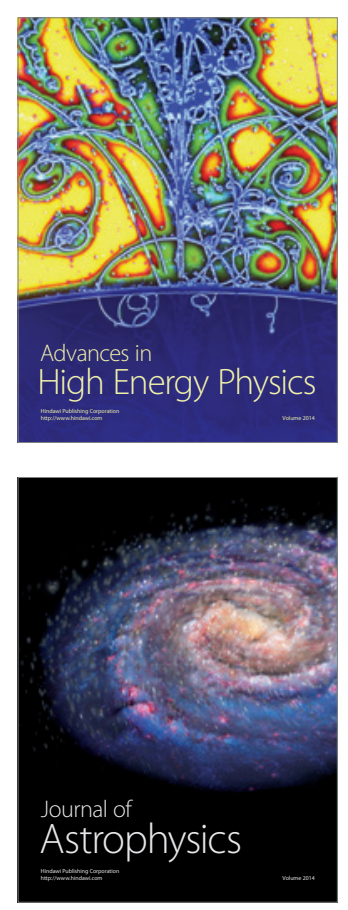
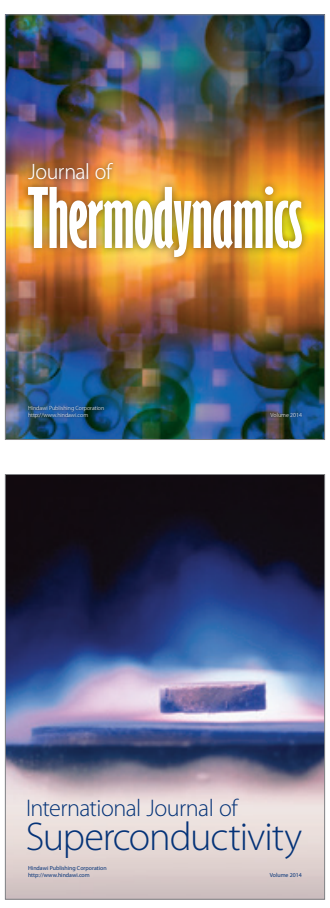
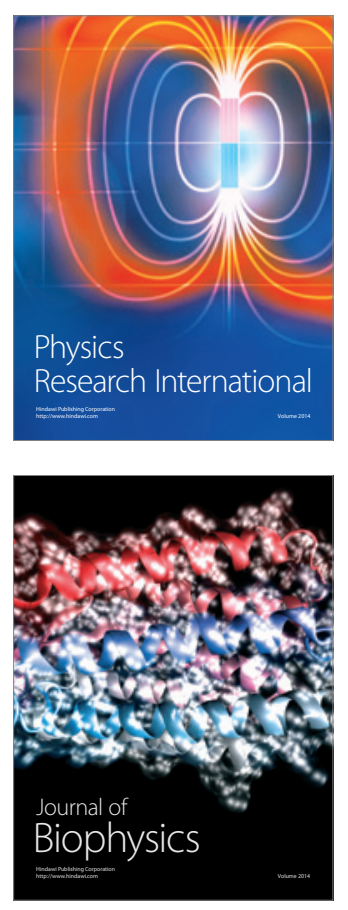
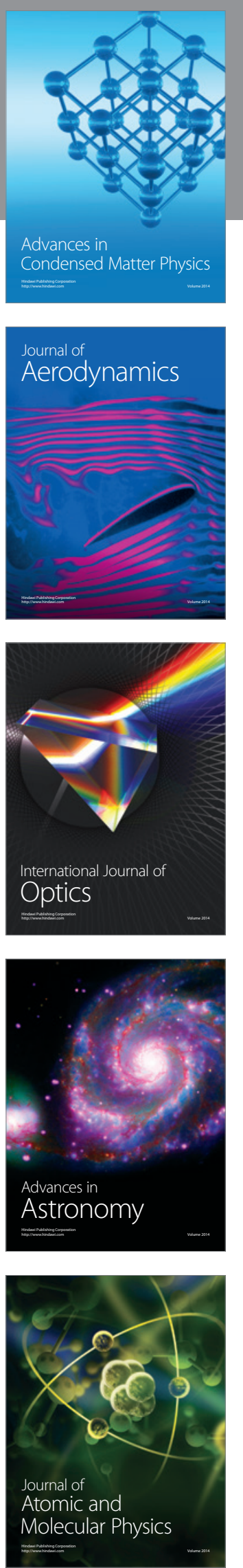